\title{
Single Nucleotide Polymorphisms in Linkage Disequilibrium with the Male-fertility Restoration (Ms) Locus in Open-pollinated and Inbred Populations of Onion
}

\author{
Michael J. Havey ${ }^{1}$ \\ Vegetable Crops Unit, Agricultural Research Service, U.S. Department of Agriculture, Department of \\ Horticulture, 1575 Linden Drive, University of Wisconsin, Madison, WI 53706
}

\begin{abstract}
Additional Index words. Allium cepa, cytoplasmic male sterility, hybrid production, marker-assisted selection
Abstract. Maintainer lines are used to seed propagate male-sterile lines for the development of hybrid onion (Allium cepa L.) cultivars. The identification of maintainer lines would be more efficient with molecular markers distinguishing genotypes at the nuclear male-fertility restoration $(M s)$ locus. $M s$ has been mapped to chromosome 2 of onion and linked genetic markers identified. However, linkages between these markers and $M s$ were detected using $\mathrm{F}_{2}$ or $\mathrm{BC}_{1}$ families at maximum linkage disequilibrium and, for many markers, their efficacy to predict genotypes at $M s$ in onion populations at or near linkage equilibrium remains unknown. In this research, near isogenic lines homozygous-dominant and -recessive at $M s$ were developed and screened for 930 single nucleotide polymorphisms (SNPs). Three SNPs tightly linked on chromosome 2 remained in linkage disequilibrium with genotypes at Ms among randomly selected plants from three open-pollinated populations of onion as well as among a collection of inbred lines. These SNPs should be useful for selection of the recessive $m s$ allele to aid in the development of maintainer lines for hybrid onion development.
\end{abstract}

Hybrid onion seed is produced using cytoplasmic-genic male sterility (CMS) systems. The most commonly used source of CMS in onion is conditioned by male-sterile (S) cytoplasm (Havey, 1993). For plants possessing $\mathrm{S}$ cytoplasm, a single nuclear locus $(M s)$ controls male-fertility restoration; a dominant allele at $M s$ conditions male fertility, whereas plants homozygous-recessive at $M s$ are male-sterile (Jones and Clarke, 1943). Plants possessing normal (N) cytoplasm are always male-fertile regardless of their genotype at Ms. Malesterile inbred lines are seed-propagated by crossing male-sterile plants (S msms) with maintainer plants that possess $\mathrm{N}$ cytoplasm and the homozygous-recessive genotype at Ms (Jones and Davis, 1944). Therefore, a main goal of hybrid onion breeding programs is to identify superior maintainer ( $\mathrm{N} \mathrm{msms}$ ) lines to seed-propagate male-sterile lines for hybrid development. The identification of maintainer lines can be expensive and time-consuming because of the biennial generation time of onion and the relatively high cost of using insects for crossing (Pike, 1986). To identify maintainers, male-sterile (S msms) plants are crossed with male-fertile ( $\mathrm{S} M s-$ or $\mathrm{N}-$ ) plants. The male fertility of progenies from the male-sterile parent is then established. If all testcross progenies are male-fertile, the male parent was homozygous-dominant at $M s$. If the progenies are all male-sterile, the male parent must be $\mathrm{N}$ msms. Finally, if the testcross progenies are segregating for male fertility, the male parent must be heterozygous at $M s$. Plants that are identified as

Received for publication 11 Apr. 2013. Accepted for publication 22 May 2013. I gratefully acknowledge the support of grant number 2008-51180-04875 from the USDA Specialty Crops Research Initiative.

Names are necessary to report factually on available data; however, the U.S. Department of Agriculture (USDA) neither guarantees nor warrants the standard of the product, and the use of the name by USDA implies no approval of the product to the exclusion of others that may also be suitable.

${ }^{1}$ Corresponding author. E-mail: mjhavey@wisc.edu.
$\mathrm{N} m s m s$ or $\mathrm{N} M s m s$ can be then used to develop maintainer lines for backcrossing to sources of S cytoplasm.

Numerous molecular markers distinguishing $\mathrm{N}$ and $\mathrm{S}$ cytoplasms of onion have been developed (Engelke et al., 2003; Havey, 1993, 1995; Kim et al., 2009; Sato, 1998). Identification of molecular markers tightly associated with the nuclear $M s$ locus would greatly aid the development of maintainer lines. These markers would allow breeders to identify plants carrying the recessive $m s$ allele early during the first year and carry forward for testcrossing only those plants with a higher probability of possessing recessive allele(s) at $M s$. We previously reported that the genomic region carrying a restriction fragment length polymorphism (RFLP) revealed by cDNA AOB272 is tightly linked $(0.9 \mathrm{cM})$ to $M s$ and converted this marker to a polymerase chain reaction (PCR)-based polymorphism (Gökçe et al., 2002). Others have either converted our previously described markers to PCR-detectable polymorphisms (Bang et al., 2011) or developed new markers linked to Ms (Huo et al., 2012; Yang et al., 2012). Although these markers are useful for selection of maintainer lines after crossing (i.e., at maximum linkage disequilibrium), they may not be in linkage disequilibrium with $M s$ among plants from open-pollinated (OP) populations (Gökçe and Havey, 2002). Yang et al. (2012) identified two markers linked to the $M s$ locus that were consistent across a sample of inbreds and hybrids; however, they proposed that their markers will be useful to genotype plants from OP populations without actually testing their use. In this research, I identified SNPs that are in linkage disequilibrium with alleles at the $M s$ locus across OP and inbred populations of onion.

\section{Materials and Methods}

Near-isogenic lines (NILs) differing for genotypes at $M s$ were developed by self-pollinating individual plants from the 
OP population 'Sapporo-Ki' (SK) and at the same time testcrossing to the male-sterile line 'MSU611-1AxMSU611B'. Pollinations were completed using flies (Jones and Emsweller, 1934). At least 20 progenies from each testcross family were scored in the field for male-fertility restoration over 2 to 4 years. $S_{1}$ progenies that were heterozygous at $M s$ (i.e., testcross family segregated for male-fertility restoration) were again self-pollinated and testcrossed to MSU611-1AxMSU611B. This process was repeated through the $\mathrm{S}_{3}$ generation, selecting in each generation single heterozygous plants for self-pollination and testcrossing. Two full-sib $\mathrm{S}_{3}$ progenies were identified as heterozygous at $M s$ and were self-pollinated to produce the $\mathrm{S}_{4}$ generation. $\mathrm{S}_{4}$ progenies were then identified as homozygous-dominant or -recessive at the $M s$ locus and were separately intercrossed to produce $\mathrm{S}_{4}$ NILs that are $\mathrm{N} M S M S$ vs. N msms. DNA was isolated from these two NILs as described by Bark and Havey (1995). A total of 930 SNPs were scored between the two NIL populations using the KASPar assay (LGC Genomics, Beverly, MA) as previously reported by Duangjit et al. (2013).

Individual plants from OP populations 'Brigham Yellow Globe' (BYG), 'Mountain Danvers' (MD), and SK were randomly selected and paired with male-sterile lines. Pollinations were completed using flies and seed was harvested separately from the male-fertile ( $\mathrm{S}_{1}$ families) and male-sterile (testcross families) plants. At least 20 progenies from each testcross family were evaluated over at least 2 years and scored as male-fertile (male parent was $M s M s$ ), segregating for male fertility (male parent Msms) or male-sterile (male parent $\mathrm{msms}$ ) as previously reported by Gökçe and Havey (2002). DNA was isolated as described by Bark and Havey (1995) from at least 25 plants from each $S_{1}$ family and a collection of inbred lines developed and released by U.S. publicsector researchers (Table 1) and genotyped for SNPs polymorphic between the two NILs using KASPar (Duangjit et al., 2013).

\section{Results and Discussion}

Testcrosses of the two NILs from SK to male-sterile MSU611-1AxMSU611B revealed 100\% male-fertile and male-sterile testcross progenies, confirming that the NILs are homozygous-dominant and -recessive at the $M s$ locus, respectively. Of 930 SNPs, 29 remained polymorphic between the NILs (Table 2) and mapped to chromosomes 1, 2, 4, 5, and 8 (Duangjit et al., 2013). SNPs on chromosomes 1, 4, and 8 were located at the ends of linkage groups (Duangjit et al., 2013) and may have remained heterozygous between the two NILs as a result of reduced crossing over. The NILs were heterozygous for numerous SNPs on chromosomes 2 and 5 (Table 2).

The $M s$ locus has been mapped to chromosome 2 and showed tight linkage to an RFLP revealed by cDNA clone AOB272 (Gökçe et al., 2002). SNPs isotig34671_610, isotig30856_1351, isotig29186_1830, isotig17237_4883, isotig28524_1455, isotig39878_664, and isotig29609_387 mapped to the same region on chromosome 2 as AOB272 in either or both the BYG15$23 \times$ AC43 or OH1 $\times 5225$ families (Duangjit et al., 2013). To estimate the degree of linkage disequilibrium with $M s$, DNAs from 148 plants from OP populations BYG (31), MD (54), and SK (63) were genotyped for the 29 SNPs. These plants had been previously genotyped at $M s$ (Gökçe and Havey, 2002) and the estimated allelic frequencies combined across all three populations for $M s$ and $m s$ were 0.59 and 0.41 , respectively. Because these three onion populations have been maintained by open pollination, only markers very near $M s$ should remain in linkage disequilibrium. As expected, all SNPs on chromosomes $1,4,5$, and 8 and many of those on chromosome 2 were over $30 \%$ recombinant between genotypes at $M s$ (Table 2). Importantly three SNPs on chromosome 2 [isotig34671_610, isotig30856_1351, and isotig29186_1830 (Table 3)] remained in disequilibrium with $M s$ across the three OP onion populations, with $11.0 \%, 13.0 \%$, and $15.7 \%$ recombinant gametes from this random sample of plants (Table 2). No recombination among these SNPs was observed in segregating families from BYG15$23 \times$ AC43 and OH1 $\times 5225$ (Duangjit et al., 2013). These three SNPs were also consistent for genotypes at $M s$ across a collection of pubic-sector inbred lines of onion, although there was recombination detected in $\mathrm{B} 1828 \mathrm{~B}, \mathrm{MSU} 8155 \mathrm{~B}$, and W52 for SNP isotig34671_610 (Table 1). Putative annotations of and

Table 1. Genotypes at the nuclear male-fertility restoration locus $(M s)$ and single nucleotide polymorphisms (SNPs) among public-sector onion inbreds. $^{\mathrm{z}}$

\begin{tabular}{|c|c|c|c|c|c|}
\hline \multirow[b]{2}{*}{ Inbred } & \multirow[b]{2}{*}{ Source $^{y}$} & \multirow[b]{2}{*}{ Genotype at $M s$} & \multicolumn{3}{|c|}{ SNP genotype ${ }^{x}$} \\
\hline & & & isotig34671_610 & isotig30856_1351 & isotig29186_1830 \\
\hline YB986A & USDA & $m s m s$ & $\mathrm{~T}: \mathrm{T}$ & $\mathrm{A}: \mathrm{A}$ & $\mathrm{G}: \mathrm{G}$ \\
\hline $\mathrm{B} 1750 \mathrm{~A}$ & USDA & msms & $\mathrm{T}: \mathrm{T}$ & $\mathrm{A}: \mathrm{A}$ & $\mathrm{G}: \mathrm{G}$ \\
\hline B1789B & USDA & msms & $\mathrm{T}: \mathrm{T}$ & A:A & $\mathrm{G}: \mathrm{G}$ \\
\hline B1794B & USDA & $m s m s$ & $\mathbf{T}: \mathbf{C}$ & A:A & $\mathrm{G}: \mathrm{G}$ \\
\hline B1828B & USDA & $m s m s$ & $\mathbf{T}: \mathbf{C}$ & $\mathrm{A}: \mathrm{A}$ & $\mathrm{G}: \mathrm{G}$ \\
\hline $\mathrm{B} 2215 \mathrm{C}$ & USDA & MSms & $\mathrm{T}: \mathrm{C}$ & G:A & G:A \\
\hline MSU2399B & MSU & $m s m s$ & $\mathrm{~T}: \mathrm{T}$ & A:A & $\mathrm{G}: \mathrm{G}$ \\
\hline MSU611-1B & MSU & $m s m s$ & $\mathrm{~T}: \mathrm{T}$ & $\mathrm{A}: \mathrm{A}$ & $\mathrm{G}: \mathrm{G}$ \\
\hline MSU8155B & MSU & $m s m s$ & T:C & A:A & $\mathrm{G}: \mathrm{G}$ \\
\hline W101B & UW & $m s m s$ & $\mathrm{~T}: \mathrm{T}$ & $\mathrm{A}: \mathrm{A}$ & $\mathrm{G}: \mathrm{G}$ \\
\hline W205B & UW & $m s m s$ & $\mathrm{~T}: \mathrm{T}$ & A:A & $\mathrm{G}: \mathrm{G}$ \\
\hline
\end{tabular}

${ }^{\mathrm{z}} \mathrm{SNP}$ genotypes shown in bold indicate that recombination was detected between the SNP and the recessive $m s$ allele.

${ }^{\mathrm{y}} \mathrm{USDA}=\mathrm{U} . \mathrm{S}$. Department of Agriculture, Beltsville, MD; MSU = Michigan State University, East Lansing; UW = University of Wisconsin, Madison.

${ }^{\mathrm{x}}$ Sequences flanking SNPs and primers used for genotyping were reported by Duangit et al. (2013). 
Table 2. Percent recombination detected between the recessive $m s$ allele and single nucleotide polymorphisms (SNPs) among random plants from three open-pollinated populations of onion.

\begin{tabular}{|c|c|c|c|}
\hline $\mathrm{SNP}^{\mathrm{z}}$ & Recombination (\%) & $\begin{array}{l}\text { Frequency of nucleotide in coupling } \\
\text { phase with recessive } m s \text { allele }\end{array}$ & Chromosome no. \\
\hline isotig34671_610 & 11.0 & $\mathrm{~T}(0.37)$ & 2 \\
\hline isotig30856_1351 & 13.0 & $\mathrm{~A}(0.46)$ & 2 \\
\hline isotig29186_1830 & 15.7 & $\mathrm{G}(0.45)$ & 2 \\
\hline isotig35505_474 & 30.0 & $\mathrm{G}(0.43)$ & Not mapped \\
\hline isotig33680_1027 & 32.5 & $\mathrm{~T}(0.45)$ & 5 \\
\hline isotig17237_4883 & 34.6 & $\mathrm{~T}(0.44)$ & 2 \\
\hline isotig30879_537 & 35.5 & $\mathrm{G}(0.55)$ & 2 \\
\hline isotig32346_843 & 37.5 & $\mathrm{~A}(0.47)$ & Not mapped \\
\hline isotig30110_847 & 37.8 & $\mathrm{~A}(0.70)$ & Not mapped \\
\hline isotig28524_1455 & 38.0 & $\mathrm{G}(0.64)$ & 2 \\
\hline isotig25881_1343 & 38.1 & $\mathrm{C}(0.40)$ & 5 \\
\hline contig00676_1004 & 38.8 & $\mathrm{~A}(0.48)$ & 1 \\
\hline isotig36793_769 & 39.0 & $\mathrm{~A}(0.59)$ & 4 \\
\hline isotig27259_617 & 40.5 & $\mathrm{C}(0.70)$ & 1 \\
\hline isotig32358_978 & 40.6 & $\mathrm{C}(0.58)$ & Not mapped \\
\hline isotig34668_1112 & 41.0 & $\mathrm{G}(0.63)$ & Not mapped \\
\hline isotig42686_290 & 41.3 & $\mathrm{~T}(0.66)$ & 8 \\
\hline isotig39878_664 & 41.8 & $\mathrm{C}(0.66)$ & 2 \\
\hline isotig35125_241 & 42.0 & $\mathrm{C}(0.57)$ & 5 \\
\hline isotig32712_566 & 43.1 & $\mathrm{~A}(0.62)$ & 3 \\
\hline isotig15820_1734 & 43.2 & $\mathrm{~A}(0.66)$ & Not mapped \\
\hline isotig29167_1843 & 44.8 & $\mathrm{~T}(0.61)$ & 5 \\
\hline isotig26178_547 & 45.5 & $\mathrm{G}(0.67)$ & 5 \\
\hline isotig29102_2197 & 45.5 & $\mathrm{~A}(0.81)$ & 1 \\
\hline isotig34751_952 & 46.9 & $\mathrm{~A}(0.68)$ & 5 \\
\hline isotig31622_435 & 49.0 & $\mathrm{~A}(0.78)$ & Not mapped \\
\hline isotig29609_387 & 50.3 & $\mathrm{~T}(0.74)$ & 2 \\
\hline isotig37821_673 & 52.4 & $\mathrm{G}(0.82)$ & 4 \\
\hline isotig12617_836 & 56.5 & $\mathrm{G}(0.79)$ & 2 \\
\hline
\end{tabular}

${ }^{\mathrm{z}}$ Names and sequences flanking SNPs and primers used for amplifications were reported by Duangjit et al. (2013).

sequences flanking the three cDNAs in linkage disequilibrium with $M s$ are listed in Table 3. Although isotig30856_1351 shows significant similarity to pentatricopeptide repeat-containing proteins, a class of nuclear genes often conditioning malefertility restoration (Schmitz-Linneweber and Small, 2008), recombination between the SNP in this cDNA and $M s$ was detected (Table 2).

The SNPs listed in Table 3 must be tightly associated with the $M s$ locus to remain in linkage disequilibrium in OP populations and should be useful for selecting plants carrying the recessive $m s$ allele for development of maintainer lines. However, because recombination between these SNPs and the Ms locus was detected among the OP and inbred populations, the success of these SNPs to predict genotypes at $M s$ will vary among onion populations. I recommend that an onion population be crossed with S-cytoplasmic, male-sterile line(s) and testcross seed evaluated for male-fertility restoration. Once it is established that the recessive $m s$ allele is present in the population, individual

Table 3. Annotations and complementary (c) DNA sequences flanking single nucleotide polymorphisms (SNPs) tightly associated with the malefertility restoration $(M s)$ locus of onion. ${ }^{z}$

\begin{tabular}{lcc}
\hline Onion cDNA & Annotation & Sequence $^{\mathrm{y}}$ \\
\hline isotig34671_610 & Auxin-induced protein & TGCAGCAACTGTCAAANATTGGATCAATCCGAAAAAGCAAGTGAAGGC \\
& & TGTTACAGAAAG[C/T]CTGGCTGGATACTGCTTTAGCACTGGAACCTG \\
isotig30856_1351 & AAGAACCATCCATCCAGACCAGGCGAGA \\
& repeat-containing protein & GTTGAAGCACTATGGATGCATGGCTGATCTGTATGGACGGTCTGGATTG \\
& GTTGATGAGGC[G/A]aTGGAGATGATAGAAGGGATGCCGATGAAGGC \\
isotig29186_1830 & RNA polymerase II C-terminal & GAATGATCCGAGTAAGAACAATGTTACGATCTGACAATCGAATAACCGG \\
& domain phosphatase-like 2 & ACGAGAAGCAC[A/G]GgTTTGTGCACCAGGTAATTGCAAGACTTGTTCG \\
& & TTTTGTGCAGATACTGGCATCCCATT \\
\hline
\end{tabular}

${ }^{\mathrm{z}}$ Polymorphic nucleotides are shown in brackets. These sequences were previously reported by Duangjit et al. (2013) and are included here for convenience only.

${ }^{\mathrm{y}}$ Lower case letters indicate nucleotides with lower quality $(<\mathrm{Q} 40)$ calls as determined by Newbler software (Duangjit et al., 2013). 
plants should be genotyped for isotig34671_610, isotig30856_1351, and isotig29186_1830 to determine if these SNPs are polymorphic, and to select individual plants carrying the SNP allele(s) putatively in coupling phase with the recessive $m s$ allele (Table 2). If these markers remained in linkage disequilibrium with the $M s$ locus in the population, discarding plants with SNP alleles in coupling phase with the dominant $M s$ allele should allow for efficient development of maintainer lines from selected plants or increase the frequency of the recessive $m s$ allele in progenies from intercrossing among the selected plants.

\section{Literature Cited}

Bang, H., D.Y. Cho, K.S. Yoo, M.K. Yoon, B.S. Patil, and S. Kim. 2011. Development of simple PCR-based markers linked to the $M s$ locus, a restorer-of-fertility gene in onion (Allium cepa L.). Euphytica 179:439-449.

Bark, O.H. and M.J. Havey. 1995. Similarities and relationships among open-pollinated populations of the bulb onion as estimated by nuclear RFLPs. Theor. Appl. Genet. 90:607-614.

Duangjit, J., B. Bohanec, A.P. Chan, C.T. Town, and M.J. Havey. 2013. Transcriptome sequencing to produce SNP-based genetic maps of onion. Theor. Appl. Genet. doi: 10.1007/s00122-0132121-x.

Engelke, T., D. Terefe, and T. Tatlioglu. 2003. A PCR-based marker system monitoring CMS-(S), CMS-(T) and (N)-cytoplasm in the onion (Allium cepa L.). Theor. Appl. Genet. 107:162-167.

Gökçe, A.F. and M.J. Havey. 2002. Linkage equilibrium among tightly linked RFLPs and the $M s$ locus in open-pollinated onion populations. J. Amer. Soc. Hort. Sci. 127:944-946.

Gökçe, A.F., J. McCallum, Y. Sato, and M.J. Havey. 2002. Molecular tagging of the Ms locus in onion. J. Amer. Soc. Hort. Sci. 127:576582.
Havey, M.J. 1993. A putative donor of S-cytoplasm and its distribution among open-pollinated populations of onion. Theor. Appl. Genet. 86:128-134.

Havey, M.J. 1995. Cytoplasmic determinations using the polymerase chain reaction to aid in the extraction of maintainer lines from openpollinated populations of onion. Theor. Appl. Genet. 90:263-268.

Huo, Y.M., J. Miao, B.J. Liu, Y.Y. Yang, Y.H. Zhang, and X. Wu. 2012. The expression of pectin methylesterase in onion flower buds is associated with the dominant male-fertility restoration allele. Plant Breed. 131:211-216.

Jones, H.A. and A. Clarke. 1943. Inheritance of male sterility in the onion and the production of hybrid seed. Proc. Amer. Soc. Hort. Sci. 43:189-194

Jones, H.A. and G.N. Davis. 1944. Inbreeding and heterosis and their relation to the development of new varieties of onions. U.S. Dept. Agr. Tech. Bul. No. 874.

Jones, H.A. and S.L. Emsweller. 1934. The use of flies as onion pollinators. Proc. Amer. Soc. Hort. Sci. 31:160.

Kim, S., E. Lee, D.Y. Cho, T. Han, H. Bang, B.S. Pati, Y.K. Ahn, and M. Yoon. 2009. Identification of a novel chimeric gene, orf725, and its use in development of a molecular marker for distinguishing among three cytoplasm types in onion (Allium cepa L.). Theor. Appl. Genet. 118:433-441.

Pike, L.M. 1986. Onion breeding, p. 357-394. In: Bassett, M. (ed.). Breeding vegetable crops. AVI Publ., Roslyn, NY.

Sato, Y. 1998. PCR amplification of CMS-specific mitochondrial nucleotide sequences to identify cytoplasmic genotypes of onion (Allium cepa L.). Theor. Appl. Genet. 96:367-370.

Schmitz-Linneweber, C. and I. Small. 2008. Pentatricopeptide repeat proteins: A socket set for organelle gene expression. Trends Plant Sci. 13:663-670.

Yang, Y., Y. Huo, J. Miao, B. Liu, S. Kong, L. Gao, C. Liu, Z. Wang, Y. Tahara, H. Kitano, and X. Wu. 2012. Identification of two SCAR markers co-segregated with the dominant $\mathrm{Ms}$ and recessive $\mathrm{ms}$ alleles in onion (Allium cepa L.). Euphytica 190:267-277. 